\title{
The computational complexity of boundedly rational choice behavior
}

\author{
Thomas Demuynck*
}

July 30, 2010

\begin{abstract}
This research examines the computational complexity of two boundedly rational choice models that use multiple rationales to explain observed choice behavior. First, we show that the notion of rationalizability by $K$ rationales as introduced by Kalai, Rubinstein, and Spiegler (2002) is $\mathcal{N} \mathcal{P}$-complete for $K$ greater or equal to two. Second, we show that the question of sequential rationalizability by $K$ rationales, introduced by Manzini and Mariotti (2007), is $\mathcal{N} \mathcal{P}$-complete for $K$ greater or equal to three if choices are single valued, and that it is $\mathcal{N} \mathcal{P}$-complete for $K$ greater or equal to one if we allow for multi-valued choice correspondences. Motivated by these results, we present two binary integer feasibility programs that characterize the two boundedly rational choice models and we compute their power. Finally, by using results from descriptive complexity theory, we explain why it has been so difficult to obtain 'nice' characterizations for these models.
\end{abstract}

JEL Classification: C60, D03, C63

Keywords: boundedly rational choice, rationalization by multiple rationales, sequential rationalization, $\mathcal{N} \mathcal{P}$-completeness, integer programming, descriptive complexity.

\section{Introduction}

Neoclassical rational choice theory departs from the assumption that a decision maker chooses among the available alternatives the ones that are highest ranked according to his/her preference relation. This preference relation is assumed to be complete, transitive, stable over time and stable across different choice environments. The neoclassical model is not only convenient from a theoretical perspective but it also implies strong and easily verifiable testable implications; e.g. Richter (1966)'s congruence condition. Unfortunately, its

${ }^{*}$ Center for Economic Studies, Catholic University of Leuven, E.Sabbelaan 53, B-8500 Kortrijk, Belgium. email: thomas.demuynck@kuleuven-kortrijk.be. I gratefully acknowledge the Fund for Scientific Research - Flanders (FWO-Vlaanderen) for my postdoctoral fellowship. 
testable implications are frequently rejected by empirical research. A first kind of rejection relates to the violation of transitivity. Cyclical choice behavior has been observed in, for example, Tversky (1969); Loomes, Starmer, and Sugden (1991); Loomes and Taylor (1992) and Roelofsma and Read (2000). A second kind of refutation pertains to the violation of contraction consistency (or independence of irrelevant alternatives) which requires that the chosen element from a set is also chosen from every subset that contains it (e.g. Seidl and Traub (1996) and Kroll and Vogt (2008)). A violation of contraction consistency leads to, so called, menu-dependent or context dependent choice behavior. ${ }^{1}$

As an answer to these empirical findings several alternative boundedly rational choice models have been developed. Boundedly rational choice models explain choice behavior by providing a more realistic and a more explicit description of how a decision maker actually makes choices. An interesting collection of these boundedly rational choice models rationalize choice behavior by making use of multiple rationales (preferences). In this research, we focus on two popular models from this collection: the model of choice by multiple rationales, introduced by Kalai, Rubinstein, and Spiegler (2002), and the model of sequential choice by multiple rationales from Manzini and Mariotti (2007). We derive the computational complexity of these two models and we provide a binary integer programming approach that enables us to verify whether a choice function can be rationalized by these models. Finally, we discuss the theoretical implications of our results. The remaining part of this introduction motivates the two models and summarizes our main contributions.

Boundedly rational choice using multiple rationales: The first boundedly rational choice model in this research is the model of choice by multiple rationalizations, introduced by Kalai, Rubinstein, and Spiegler (2002). The idea behind this model is that choices are menu dependent. More formally, the model of choice by multiple rationales is determined by a collection of rationales (preference relations). This collection is said to rationalize the choice behavior of the decision maker if each observed choice is maximal for at least one rationale in the collection. Kalai, Rubinstein, and Spiegler (2002) motivate their model using the example of Luce and Raiffa (1957)'s dinner. In this example, a customer chooses a meal from a menu in a restaurant. His choice is chicken when the menu provides the choice between steak tartare or chicken. On the other hand, the customer chooses steak tartare when the menu provides the choice between steak tartare, chicken or frog's legs. The choice is explained by the fact that the presence of frog's legs (something that is difficult to prepare) is an indication of the high quality of the chef. In addition, expertise is necessary to make a good steak tartare. As such, the presence of frog's legs on the menu conveys information about the quality of the other available alternatives, a clear example of context dependent choice. Kalai, Rubinstein, and Spiegler (2002) provide several results concerning the minimal number of rationales needed in order to rationalize a given choice function. Recently, Apesteguia and Ballester (2010) showed that computing this minimal number of rationales is a difficult problem $(\mathcal{N} \mathcal{P}$-complete). We will come back to this

\footnotetext{
${ }^{1}$ Interestingly, under the full domain assumption, contraction consistency implies acyclic choice behavior (see, for example, Suzumura (1983)).
} 
result in section 3 .

The second boundedly rational model is the model of sequential choice by multiple rationales from Manzini and Mariotti (2007). In this model, choices are made by solving a sequence of intermediate smaller choices. In particular, the decision maker is endowed with a fixed number of rationales (preferences) which are sequentially applied to narrow down the remaining set of alternatives. Manzini and Mariotti (2007) give the example of an arbitrator who has to pick one out of three available alternatives $a, b$ or $c$. Assume that $c$ is Pareto superior to $a$ while $a$ seems fairer than $b$ and $b$ seems fairer than $c$. If the arbitrator first chooses accordingly to the Pareto criterion and then according to the fairness criterion he will choose $c$ from the choice set $\{a, c\}, a$ from the choice set $\{a, b\}$ and $b$ from the choice set $\{b, c\}$, invoking cyclical (intransitive) behavior. Manzini and Mariotti (2007) characterize the choice functions that are sequentially rationalizable by two and three rationales.

Contribution: Recently, there has been a growing interest in the application of computational complexity theory to economic interesting problems (see, among many others, Gilboa and Zemel (1989); Chu and Halpern (2001); Cechlarova and Hajdukova (2002); Fang, Zhu, Cai, and Deng (2002); Woeginger (2003); Baron, Durieu, Haller, and Solal (2004); Baron, Durieu, Haller, Savani, and Solal (2008); Brandt and Fisher (2008); Conitzer and Sandholm (2008); Kalyanaraman and Umans (2008); Procaccia and Rosenschein (2008); Cherchye, Demuynck, and De Rock (2009); Galambos (2009); Hudry (2009); Brandt, Fisher, Harrenstein, and Mair (2010); Talla Nobibon, Cherchye, De Rock, Sabbe, and Spieksma (2010) and Apesteguia and Ballester (2010)). However, as noted by Apesteguia and Ballester (2010), there is relatively little work that applies the insights of computational complexity theory to (rational) choice theory. In this research we partially fill this gap. We provide the computational complexity of the two aforementioned boundedly rational choice models. We show that the question of rationalizability by multiple rationales is $\mathcal{N} \mathcal{P}$-complete as soon as the set of rationales has size greater or equal to two. Further we show that, for single valued choices, the question of sequential rationalization by multiple rationales is $\mathcal{N} \mathcal{P}$-complete as soon as the number of rationales is greater or equal to three. Finally, we show that for multi-valued choice correspondences sequential rationalizability is $\mathcal{N} \mathcal{P}$-complete as soon as there is a single rationale.

Our computational complexity results have both empirical and theoretical consequences. From an empirical point of view, the fact that the verification of a certain choice model is $\mathcal{N} \mathcal{P}$-complete shows that empirical refutation or acceptance of these models might be extremely difficult. At present, the fastest algorithm to solve any $\mathcal{N} \mathcal{P}$-complete problem has exponential worst time complexity. In order to deal with this issue we suggest to use a binary integer programming procedure (BIP) to verify the boundedly rational choice models. This approach has several advantages. First of all, it is a widely accepted, widely available and well known approach to handle $\mathcal{N} \mathcal{P}$-complete problems. A second argument pro our BIP approach is that it provides a very flexible framework to modify certain aspects of our problem. In section 3 we will show how we can modify the framework to 
include additional assumptions on the rationales, like, for example, transitivity, acyclicity, connectedness, etc. We illustrate the usefulness of our approach by computing the power (i.e. the probability of rejecting random choice behavior) of the two boundedly rational choice models. We also discuss recovery and goodness-of-fit measurement.

Our $\mathcal{N} \mathcal{P}$-completeness results have also theoretical consequences. For these we draw on the theory of descriptive complexity. Descriptive complexity theory studies the relationship between the computational complexity of a certain decision problem and the logical language that is necessary to characterize the set of solutions for this decision problem. Using the results from this literature, we explain why it has been so difficult to obtain 'nice' characterizations for the two boundedly rational choice models. ${ }^{2}$ Finally, we show that these findings are not limited to the models discussed in this research. In particular, we link it to the the problem of maximal element rationalizability, introduced by Bossert, Sprumont, and Suzumura (2005).

The next section introduces notation and presents our computational complexity results. Section three presents the binary programming characterization. Section four discusses the descriptive complexity implications of our results and section five presents a short conclusion.

\section{Notation and Definitions}

Choice by multiple rationales: Consider a finite set of alternatives $X$ with cardinality $n$. A choice function $c$ is a correspondence from a collection $\Sigma \subseteq 2^{X}-\{\emptyset\}$ to $2^{X}-\{\emptyset\}$ such that for all $A \in \Sigma, c(A) \subseteq A$ and $c(A) \neq \emptyset$. We denote by $m$ the number of choice sets in $\Sigma$. In this paper we do not impose that the universal domain assumption holds, i.e. we do not require that $\Sigma$ contains all nonempty subsets of $X$. Although this assumption is frequently used in choice theoretic research, it is not convenient for the evaluation of computational complexity issues as it implies that the size of the choice domain $m$ is exponential in the number of alternatives $n$. For example, with 15 alternatives the universal domain assumption requires that $c$ is defined on a domain of 32.753 choice sets, clearly beyond any practical relevance.

We say that the domain $\Sigma$ is binary if it includes all 2 element subsets of $X$ : for all $x, y \in X,\{x, y\} \in \Sigma$. The choice function $c$ is single valued if for all $A \in \Sigma, c(A)$ contains a single alternatives.

For a set $A \subseteq X$ and an binary relation $P \subset X \times X$, we denote by $\mathcal{M}(A, P)$ the set of $P$-maximal elements of $A$. This is the collection of elements in $A$ that are not dominated by any other element in $A$ according to the relation $P$.

$$
\mathcal{M}(A, P)=\{x \in S \mid \nexists y \in A,(y, x) \in P\} .
$$

If the relation $P$ is connected, i.e. for all $x, y$ in $X$ with $x \neq y$, either $(x, y) \in P$ or $(y, x) \in P$, the set of $P$-maximal elements coincides with the set of $P$-greatest elements

\footnotetext{
${ }^{2}$ In Section 5 , it will become clear what we mean by a 'nice' characterization.
} 
$\mathcal{G}(A, P)$

$$
\mathcal{G}(A, P)=\{x \in A \mid \forall y \in A,(x, y) \in P\} .
$$

To introduce the concept of rationalization by multiple rationales we start from a list of connected, transitive and asymmetric relations $\left(P_{k}\right)_{k \leq K} \cdot{ }^{3}$ We say that a choice function $c$ is rationalizable by the the list $\left(P_{k}\right)_{k \leq K}$ if for every choice set $A$ in the domain $\Sigma$ there is at least one rationale $P_{k}$ for which the choice $c(A)$ is equal to the set of $P$-greatest elements in $A$. Formally:

Definition 1 ( $K$-Rationalizable by Multiple Rationales). A list of $K$ asymmetric, transitive and connected relations $\left(P_{k}\right)_{k \leq K}, K$-rationalizes the choice function $c$ on the domain $\Sigma$ if for all $A \in \Sigma$ there exist at least one $k \leq K$ for which:

$$
c(A)=\mathcal{G}\left(A, P_{k}\right)
$$

Our second model of choice behavior is sequential rationalizability. This model departs from a list of asymmetric relations $P_{1}, \ldots, P_{k}$ and applies each of these relations sequentially, eliminating in each round the alternatives that are dominated.

Definition 2 ( $K$-Sequential Rationalizability). A list of $K$ asymmetric relation $\left(P_{k}\right)_{k \leq K}$, $K$-sequentially rationalizes the choice function $c$ on the domain $\Sigma$ if and only if for all $A \in \Sigma$, defining recursively:

$$
\begin{gathered}
M_{0}(A)=A, \\
M_{k}(A)=\mathcal{M}\left(M_{k-1}(A), P_{k}\right), k=1, \ldots, K,
\end{gathered}
$$

we have

$$
c(A)=M_{K}(A)
$$

The intuition is the following. First one considers a sequence of binary relations $P_{1}, \ldots, P_{K}$. Then for all choice sets $A$, in the first round of the decision process one computes the undominated elements of $A$ according to the relation $P_{1}, M_{1}(A)=\mathcal{M}\left(A, P_{1}\right)$. Next one looks for the undominated elements of $M_{1}(A)$ according to the relation $P_{2}$. $M_{2}(A)=\mathcal{M}\left(M_{1}(A), P_{2}\right)$. In the third step, one retrieves the undominated elements of $M_{2}(A)$ according to the relation $P_{3}, M_{3}(A)=\mathcal{M}\left(M_{2}(A), P_{3}\right)$. This procedure is applied sequentially until the last set $M_{K}(A)$ is computed. Consider a choice function $c$ on a domain $\Sigma$ and a $K$-sequential rationalization $\left(P_{k}\right)_{k \leq K}$ of this choice function. If for all $A \subseteq X$, the set $M_{K}(A)$ is single valued, i.e. contains a single element, we say that $\left(P_{k}\right)_{k \leq K}$ is a single-valued $K$-sequential rationalization of $c$. Otherwise, we say that $\left(P_{k}\right)_{k \leq K}$ is a multi-valued $K$-sequential rationalization. Observe that multi-valued rationalizations do does not exclude cases for which $M_{K}(A)$ might be empty for some $A \subseteq X-\Sigma$, which can occur if one of the rationales has a cycle. Further if the domain is binary then any sequential rationalization of $c$ is single-valued if and only if $c$ is single valued.

\footnotetext{
${ }^{3}$ A relation $P$ is asymmetric if for all $x$ and $y$ in $X$ it is not the case that both $(x, y) \in P$ and $(y, x) \in P$. The relation is transitive if for all $x, y$ and $z$ in $X,(x, y) \in P$ and $(y, z) \in P$ implies $(x, z) \in P$.
} 
Computational complexity: The theory of computational complexity tries to answer how much time and how much memory space is needed to solve a particular problem. To be compact, we will only give an intuitive introduction into this theory, alas at the cost of accuracy. For a detailed introduction into the theory of computational complexity and $\mathcal{N} \mathcal{P}$-completeness in particular, we refer to the seminal work of Garey and Johnson (1979).

The primitives in the theory of computational complexity are the decision problems. Each decision problem is composed of a collection of instances, the inputs for the problem, and a 'yes' /'no' question that asks whether a particular instance satisfies a certain property. In other words, a decision problem corresponds to each of its instances either a 'yes' or a 'no' as an output depending on whether the instance satisfies the question. In this research the instances of our decision problems will always consist of the set of alternatives, a domain and a choice function:

INSTANCE: An instance is given by the triplet $(X, \Sigma, c)$ composed of a finite set of alternatives $X$ of size $n$, a collection of nonempty subsets of $X$, denoted by $\Sigma$, of size $m$ and a correspondence $c$ from $\Sigma$ to $X$ such that for all $A \in \Sigma, c(A) \subseteq A$ and $c(A) \neq \emptyset$.

The input size of the instance $(X, \Sigma, c)$ is given by the size of the set of alternatives $n$ together with the size of the domain $m .{ }^{4}$ The following three questions define our decision problems.

\section{$K$-Rationalizable by Multiple Rationales ( $K$-RMR)}

QUESTION: Given an instance $(X, \Sigma, c)$, does there exist a list of $K$ transitive, asymmetric and connected relations $\left(P_{i}\right)_{i \leq K}$ such that this list $K$-rationalizes the choice function $c ?$

\section{$K$-Sequentially Rationalizable ( $K$-SR)}

QUESTION: Given an instance $(X, \Sigma, c)$, does there exist a list of $K$ asymmetric relations $\left(P_{i}\right)_{i \leq K}$ such that this list is a (multi-valued) $K$-sequential rationalization of $c$ ?

\section{$K$-Sequential Rationalizable by a single-valued rationalization ( $K$-SRS)}

QUESTION: Given an instance $(X, \Sigma, c)$, does there exist a list of $K$ asymmetric relations $\left(P_{i}\right)_{i \leq K}$ such that this list is a single valued $K$-sequential rationalization of $c$ ?

Observe that for the problems $K$-RMR, $K$-SR and $K$-SRS, the number of rationales $K$ is given as a parameter of the problem. In other words, there are an infinite number of decision problems, one for each value of $K \in \mathbb{N}-\{0\}$.

We denote by $\mathcal{P}$ the class of decision problems that can be solved in a polynomial number of steps on a deterministic Turing machine. ${ }^{5}$ A second important class of decision problems are the decision problems that are solvable in nondeterministic polynomial time,

\footnotetext{
${ }^{4}$ If we want to reduce the size to a single number, we can take $\max \{n, m\}$

${ }^{5}$ More precisely, there exist a polynomial function, $f$, of the input size, here $\max \{m, n\}$, such that a solution can be found in less than $f(\max \{m, n\})$ steps.
} 
denoted by $\mathcal{N} \mathcal{P}$. A decision problem belongs to the class $\mathcal{N} \mathcal{P}$ if, given a "yes" instance to the question there exist at least one polynomial-time certificate such that in polynomial time, a deterministic Turing machine accepts the certificate as proof for a 'yes' answer and given a 'no' instance of the problem, no certificate is ever accepted. For example, $K$-RMR is in the class $\mathcal{N} \mathcal{P}$ if, for any list of $K$ asymmetric, transitive and connected relations $\left(P_{k}\right)_{k \leq K}$ that $K$-rationalizes the instance $(X, \Sigma, c)$, there exists a polynomial time algorithm that provides a proof that $\left(P_{k}\right)_{k \leq K}$ is indeed a $K$-rationalization of $c$. A decision problem which is at least as hard (as difficult) as any other problem in $\mathcal{N} \mathcal{P}$ is called $\mathcal{N} \mathcal{P}$ hard. A decision problem is said to be $\mathcal{N} \mathcal{P}$-complete if it is both $\mathcal{N} \mathcal{P}$-hard and in $\mathcal{N} \mathcal{P}$. In other words, an $\mathcal{N} \mathcal{P}$-complete problem is at least as difficult as any other problem from the class $\mathcal{N} \mathcal{P}$.

A fundamental open question in computational complexity (and in all of mathematics) is whether the class of decision problems in $\mathcal{P}$ is equal to the class of decision problems in $\mathcal{N} \mathcal{P}(\mathcal{P} \stackrel{?}{=} \mathcal{N} \mathcal{P})$. By definition, we have that $\mathcal{P} \subseteq \mathcal{N} \mathcal{P}$. It is not know, at present, whether all problems in $\mathcal{N} \mathcal{P}$ can be solved in polynomial time. The general accepted belief is that $\mathcal{P} \neq \mathcal{N} \mathcal{P}$.

An $\mathcal{N} \mathcal{P}$-complete problem is among the most difficult problems in the class $\mathcal{N} \mathcal{P}$. This class contains most of the natural computable problems from the real world. Hence $\mathcal{N} \mathcal{P}$ complete problems are considered to be computationally intractable (especially for large sized problems). In fact, all known solutions to $\mathcal{N} \mathcal{P}$-complete problems have exponential worst time complexity.

In order to show that a certain decision problem is $\mathcal{N} \mathcal{P}$-complete one must demonstrate two things. First one has to demonstrate that the decision problem is in $\mathcal{N} \mathcal{P}$. Second one has to show that it is harder than any other problem in $\mathcal{N} \mathcal{P}$. The way to solve this second problem is to show that a known $\mathcal{N} \mathcal{P}$-complete problem is polynomial time reducible into the given problem. In particular, a problem $Q_{1}$ is polynomial time reducible into a problem $Q_{2}$ if (i) there exist a function $g$ which maps every instance, $I_{1}$ of $Q_{1}$ into an instance, $g\left(I_{1}\right)$ of $Q_{2}$ in such a way that the instance $I_{1}$ is a 'yes' for $Q_{1}$ if and only if $g\left(I_{1}\right)$ is a 'yes' for $Q_{2}$, and (ii) $g\left(I_{1}\right)$ can be constructed in polynomial time.

\section{Computational complexity results}

Rationalization by multiple rationales: For $K=1$, the decision problem $K$-RMR reduces to the question whether the choice function is rationalizable by a single transitive, asymmetric and connected preference relation. It is well-known that this problem can be solved in polynomial time (see for example Apesteguia and Ballester (2010)). The interesting question is whether this remains true if we consider decision problems with $K \geq 2$. Recently, Apesteguia and Ballester (2010) showed that there exists at least one $K$ such that $K$-RMR is $\mathcal{N} \mathcal{P}$-complete. In particular they showed $\mathcal{N} \mathcal{P}$-completeness of the following decision problem:

QUESTION RMR: Given an instance $(X, \Sigma, c)$ and a number $K$, can we find a list 
of $T \leq K$ transitive, asymmetric and complete relations, $\left(P_{i}\right)_{i \leq T}$ such that this list $T$ rationalizes the choice function $c$ ?

Of course, $\mathcal{N} \mathcal{P}$-completeness of the decision problem RMR does not rule out that for particular values of $K, K$-RMR might be in $\mathcal{P}$ (this is the case, for example, when $K=1$ ). Unfortunately, our first results shows that as soon as $K$ is greater or equal to two, $\mathcal{N} \mathcal{P}$-completeness prevails. For $K=2$ the proof uses a reduction from the $\mathcal{N} \mathcal{P}$-complete problem 'Monotone-not-all-equal 3SAT'. ${ }^{6}$ The proof for $K>2$ is by induction.

Proposition 1. The decision problem $K-R M R$ is $\mathcal{N} \mathcal{P}$-complete for all $K \geq 2$.

Proof. It is easy to see that $K-R M R$ is in $\mathcal{N} \mathcal{P}$ for all values of $K$. For the second part of the proof, we need to show that a known $\mathcal{N} \mathcal{P}$-complete problem is polynomial time reducible to $K$-RMR. We first consider the case of $K=2$.

The known $\mathcal{N} \mathcal{P}$-complete problem that we use is 'Monotone not all equal 3SAT' (MNAE-3SAT). It is given by the following instances and question.

\section{Not all equal 3SAT (NAE-3SAT)}

INSTANCE: A set of binary variables $x_{1}, \ldots, x_{t}$ and a collection of clauses $C_{1}, \ldots, C_{r}$. Each clause is composed of three variables. The input size is given by the numbers $t$ and $r$.

QUESTION: Does there exist an assignment to the variables $x_{1}, \ldots, x_{t}$ (either 1 or 0 ) such that each clause contains at least one variable with the value 1 and at least one variable with the value 0 .

Consider an instance of M-NAE-3SAT with a set of variables $\left\{x_{1}, \ldots, x_{t}\right\}$ and a set of clauses $\left\{C_{1}, \ldots, C_{r}\right\}$. First we create the corresponding instance of 2-RMR, i.e. set of alternatives, the choice sets and the choice function. The following defines the alternatives:

- For each variable $x_{i}(i=1, \ldots, t)$ we construct two alternatives $a_{i}$ and $\bar{a}_{i}$.

- For each clause $C_{\ell}(\ell=1, \ldots, r)$, we construct three alternatives $z_{1, \ell}, z_{2, \ell}$ and $z_{3, \ell}$.

Consider the function $f$ from the set of variables $z_{k, \ell}(k=1,2,3$ and $\ell=1, \ldots, r)$ to the set $\{1, \ldots, t\}$, such that $f\left(z_{k, \ell}\right)=i$ if and only if the $k$-th variable in clause $C_{\ell}$ is equal to $x_{i}$. Further for $k=1,2,3$ denote by $k \oplus 1$ the number $(k+1) \bmod 3$. The choice sets and the choice function is constructed in the following way:

- For each $i=1, \ldots, t$ we construct the choice set $A_{i}=\left\{a_{i}, \bar{a}_{i}\right\}$ with $c\left(A_{i}\right)=\left\{a_{i}\right\}$.

- For all $k=1,2,3$ and $\ell=1, \ldots, r$ we construct the choice set $B_{k, \ell}=\left\{a_{i}, \bar{a}_{i}, z_{k, \ell}\right\}$ where $i=f\left(z_{k, \ell}\right)$. We set $c\left(B_{k, \ell}\right)=\left\{\bar{a}_{i}\right\}$.

\footnotetext{
${ }^{6}$ Monotone-not-all-equal-3SAT can be reduced from the $\mathcal{N} \mathcal{P}$-complete problem Not-all-equal-3SAT (Garey and Johnson, 1979) by replacing all literals of the form $\left(1-x_{i}\right)$ by a variable $y_{i}$ and adding an additional clause of the form $\left\{y_{i}, x_{i}, x_{i}\right\}$.
} 
- Finally, for each $k=1,2,3, \ell=1, \ldots, r$ we construct the choice set $D_{k, \ell}=\left\{z_{k, \ell}, z_{k \oplus 1, \ell}, \bar{a}_{i}\right\}$ with $i=f\left(z_{k, \ell}\right)$. We set $c\left(D_{k, \ell}\right)=\left\{z_{k, \ell}\right\}$.

Evidently, this construction can be performed in a polynomial number of steps.

Let us first show that when this problem satisfies 2-RMR then there must be a truth assignment that satisfies M-NAE-3SAT. Let $P_{1}$ and $P_{2}$ be the two rationales that lead to a solution for 2-RMR. To each choice set in $\Sigma$, we can correspond a rationale $\left(P_{1}\right.$ or $\left.P_{2}\right)$ that rationalizes the choice made from this set. It is easy to establish, by asymmetry of $P_{1}$ and $P_{2}$, that for each $i=1, \ldots, t$ and all $z_{k, \ell}$ with $i=f\left(z_{k, \ell}\right)$, there are only two mutually exclusive configurations possible. These are listed in the following table:

\begin{tabular}{l|c|c|c} 
choice set & choice & configuration 1 & configuration 2 \\
\hline$A_{i}=\left\{a_{i}, \bar{a}_{i}\right\}$ & $\left\{a_{i}\right\}$ & $P_{1}$ & $P_{2}$ \\
$B_{k, \ell}=\left\{a_{i}, \bar{a}_{i}, z_{k, \ell}\right\}$ & $\left\{\bar{a}_{i}\right\}$ & $P_{2}$ & $P_{1}$ \\
$D_{k, \ell}=\left\{z_{k, \ell}, z_{k \oplus 1, \ell}, \bar{a}_{i}\right\}$ & $\left\{z_{k, \ell}\right\}$ & $P_{1}$ & $P_{2}$
\end{tabular}

Now, if configuration 1 prevails for $i \in\{1, \ldots, t\}$, we set $x_{i}=1$ and if configuration 2 prevails, we set $x_{i}=0$. All we need to show now is that this solution provides a 'yes' instance of M-NAE-3SAT. Assume, on the contrary, that there is a clause $C_{\ell}$ for which all variables are equal to 1 . In that case, we have that $\left(z_{1, \ell}, z_{2, \ell}\right) \in P_{1},\left(z_{2, \ell}, z_{3, \ell}\right) \in P_{1}$ and $\left(z_{3, \ell}, z_{1, \ell}\right) \in P_{1}$, contradicting acyclicity of $P_{1}$. On the other hand if all variables in $C_{\ell}$ are equal to zeros we must have that: $\left(z_{1, \ell}, z_{2, \ell}\right) \in P_{2},\left(z_{2, \ell}, z_{3, \ell}\right) \in P_{2}$ and $\left(z_{3, \ell}, z_{1, \ell}\right) \in P_{2}$, contradicting acyclicity of $P_{2}$. Conclude that M-NAE-3SAT must be satisfied.

Let us now show that any 'yes' instance of M-NAE-3SAT corresponds to a 'yes' instance of 2-RMR. Towards this end, notice that it is sufficient to to show the existence of two acyclic and asymmetric relations $P_{1}$ and $P_{2}$ such that for each choice set, $F \in \Sigma$ with $b \in c(F)$ either $(b, d) \in P_{1}$ for all $d \in F$ or $(b, d) \in P_{2}$ for all $d \in F$. These relations $P_{1}$ and $P_{2}$ can always be extended to connected, transitive and asymmetric relations in a polynomial number of steps (using for example a finite analogue of Szpilrajn's lemma (Szpilrajn, 1930)). We assign $P_{1}$ and $P_{2}$ to the choice sets in the following way, depending on the specific value of $x_{i}(i=1, \ldots, t)$ :

\begin{tabular}{l|c|c|c} 
choice set & choice & $x_{i}=1$ & $x_{i}=0$ \\
\hline$A_{i}=\left\{a_{i}, \bar{a}_{i}\right\}$ & $a_{i}$ & $P_{1}$ & $P_{2}$ \\
$B_{k, \ell}=\left\{a_{i}, \bar{a}_{i}, z_{k, \ell}\right\}$ & $\bar{a}_{i}$ & $P_{2}$ & $P_{1}$ \\
$D_{k, \ell}=\left\{z_{k, l}, z_{k \oplus 1, \ell}, \bar{a}_{i}\right\}$ & $z_{k, \ell}$ & $P_{1}$ & $P_{2}$
\end{tabular}

In other words, if $x_{i}=1$ we add the elements $\left(a_{i}, \bar{a}_{i}\right),\left(z_{k, \ell}, z_{k \oplus 1, \ell}\right)$ and $\left(z_{k, \ell}, \bar{a}_{i}\right)$ to $P_{1}$ and the elements $\left(\bar{a}_{i}, a_{i}\right)$ and $\left(\bar{a}_{i}, z_{k, \ell}\right)$ to $P_{2}$ (given that $\left.f\left(z_{k, \ell}\right)=i\right)$. On the other hand, if $x_{i}=0$ we add the elements $\left(a_{i}, \bar{a}_{i}\right),\left(z_{k, \ell}, z_{k \oplus 1, \ell}\right)$ and $\left(z_{k, \ell}, \bar{a}_{i}\right)$ to $P_{2}$ and the elements $\left(\bar{a}_{i}, a_{i}\right)$ and $\left(\bar{a}_{i}, z_{k, \ell}\right)$ to $P_{1}$ (given that $f\left(z_{k, \ell}\right)=i$ ).

We still need to show that the relations $P_{1}$ and $P_{2}$ are acyclic. Assume, on the contrary, that there exist alternatives $b_{1}, \ldots, b_{q}$ such that for each $s=1, \ldots, q-1,\left(b_{s}, b_{s+1}\right) \in P_{1}$ and $\left(b_{q}, b_{1}\right) \in P_{1}$. (The case of a cycle in the relation $P_{2}$ is similar and is left to the reader.) We consider different cases depending on the the different possible values for $b_{1}$. 
- Case 1. $b_{1}=a_{i}$ for some $i=1, \ldots, t$. Then there must exists alternatives $b_{q}$ and $b_{2}$ such that $\left(b_{q}, a_{i}\right) \in P_{1}$ and $\left(a_{i}, b_{2}\right) \in P_{1}$. This is impossible as it implies that both $x_{i}=1$ and $x_{i}=0$.

- Case 2. $b_{1}=\bar{a}_{i}$ for some $i=1, \ldots, t$. Then there must exist alternatives $b_{q}$ and $b_{2}$ such that $\left(\bar{a}_{i}, b_{2}\right) \in P_{1}$ and $\left(b_{q}, \bar{a}_{i}\right) \in P_{1}$. Again this would imply that both $x_{i}=1$ and $x_{i}=0$.

- Case 3. $b_{1}=z_{k, \ell}$ for some $k=1,2,3$ and $\ell=1, \ldots, r$. Now, $b_{2}$ cannot be equal to $\bar{a}_{i}$ because then we are back at case 2 (replacing $b_{1}$ by $b_{2}, b_{2}$ by $b_{3}$ and $b_{q}$ by $b_{1}$.). As such, the cycle under consideration must be the cycle $\left(z_{1, \ell}, z_{2, \ell}\right) \in P_{1},\left(z_{2, \ell}, z_{3, \ell}\right) \in P_{1}$ and $\left(z_{3, \ell}, z_{1, \ell}\right) \in P_{1}$. However, this implies that all variables in clause $C_{l}$ are equal to one, a contradiction.

Conclude that 2-RMR is satisfied.

Until now, we have demonstrated that 2 -RMR is $\mathcal{N} \mathcal{P}$-complete. To show that $K$-RMR is $\mathcal{N} \mathcal{P}$-complete for all $K>2$ we use an induction argument. We know that it is $\mathcal{N} \mathcal{P}$ complete for $K=2$. Assume that it is $\mathcal{N} \mathcal{P}$-complete for $K=M$ and consider the case $K=M+1$. The following constructs the instance $\left(X^{\prime}, \Sigma^{\prime}, c^{\prime}\right)$ of $(M+1)$-RMR (the instance is denoted by primes) based on the instance $(X, \Sigma, c)$ of the decision problem $M$-RMR.

- For each $x \in X$, we create an alternative $x \in X^{\prime}$. Further, we create two additional alternatives $a^{\prime}, b^{\prime} \in X^{\prime}$.

- For each $A \in \Sigma$, create the choice set $A^{\prime}=A \cup\left\{a^{\prime}\right\}$ and impose that $c^{\prime}\left(A^{\prime}\right)=c(A)$.

- Create one additional choice set $Z=X^{\prime}$ and impose that $c^{\prime}(Z)=\left\{a^{\prime}\right\}$.

Of course, the instance $\left(X^{\prime}, \Sigma^{\prime}, c^{\prime}\right)$ can be constructed in a polynomial number of steps. Now, assume that $\left(P_{i}\right)_{i \leq M}$ is a 'yes' instance of $M$-RMR. Construct the relations $P_{i}^{\prime}=$ $P_{i} \cup\left\{\left(x, a^{\prime}\right),\left(x, b^{\prime}\right) \mid x \in X^{\prime}-\left\{a^{\prime}\right\}\right\} \cup\left\{\left(b^{\prime}, a^{\prime}\right)\right\}$ and let $P_{M+1}^{\prime}$ be an arbitrary (transitive, asymmetric and connected) relation where the alternative $a^{\prime}$ is top ranked among all alternatives. It is clear to see that $\left(P_{i}^{\prime}\right)_{i \leq M+1}$ provides a 'yes' instance to the $(M+1)$-RMR problem where $P_{M+1}^{\prime}$ rationalizes the choice set $Z$ and $P_{i}^{\prime}$ rationalizes the choice set $A^{\prime}$ if and only if $P_{i}$ rationalizes the choice set $A$.

On the other hand, assume that $\left(P_{i}^{\prime}\right)_{i \leq M+1}$ is a 'yes' instance to the $(M+1)$-RMR problem. Let $P_{M+1}^{\prime}$ be the relation that rationalizes the choice function $Z$. It must be that $a^{\prime}$ is top ranked in this relation (this follows from the fact that $Z=X^{\prime}$ and $c^{\prime}(Z)=\left\{a^{\prime}\right\}$ ). For any other choice set, $A^{\prime}$, it must be that $P_{M+1}^{\prime}$ does not rationalize this set (this is because $a^{\prime}$ is in $A^{\prime}$ but not chosen). Now, Let $P_{i}(i=1, \ldots, M)$ be the relation which is equal to $P_{i}^{\prime}$ less the comparisons involving the alternative $a^{\prime}$. Evidently $P_{i}$ rationalizes the choice set $A$ if and only if $P_{i}^{\prime}$ rationalizes the choice set $A^{\prime}$. As such $\left(P_{i}\right)_{i \leq M}$ rationalizes the choice function $c$. 
Sequential rationalizability: Now, we look at the computational complexity of the decision problems $K$-SR and $K$-SRS. We first consider the decision problem with $K=1$. It is easy to see that in this case, $K$-SRS requires the rationalization $P_{1}$ to be transitive, asymmetric and connected. As such 1-SRS agrees with 1-RMR, which we know to be in $\mathcal{P}$. On the other hand, the decision problem 1 -SR is $\mathcal{N} \mathcal{P}$-complete. We prove this in proposition 7 in section 5 .

Let us now look at the decision problem 2-SRS. Manzini and Mariotti (2007) provide a characterization of 2-SRS for the case where the domain $\Sigma$ is binary.

Theorem 1 (Manzini and Mariotti (2007)). An instance $(X, \Sigma, c)$ with $\Sigma$ a binary domain is a 'yes' instance for 2-SRS if and only if it satisfies $W W E$ : $c$ is single valued and if $x=$ $c\left(S_{i}\right)$ in a class and $x=c(\{x, y\})$ then $y \neq c(R)$ for all $R \in \Sigma$ with $\{x, y\} \subset R \subseteq \bigcup_{i \leq t} S_{i}$.

It is easy to see that the verification of WWE can proceed in a polynomial number of steps. Let us now consider the case where we abandon the requirement of the binary domain. The following theorem is a slight adaptation of theorem 1 for this more general case.

Proposition 2. An instance $(X, \Sigma, c)$ is a 'yes' instance for 2-SRS if and only if it satisfies $N B$-WWE: $c$ is single-valued and for all $x$ and $y$ in $X$ and $R, T \in \Sigma$, if $x \in c\left(S_{i}\right)$ in a class and $y \in c\left(V_{i}\right)$ in a class, $R \subseteq \bigcup_{i} S_{i}$ and $T \subseteq \bigcup_{i} V_{i}$, then not $x \in c(T)$ and $y \in c(R)$.

Proof. Necessity is obvious. For sufficiency, notice that it is sufficient to demonstrate that for every instance $(X, \Sigma, c)$ that is a 'yes' instance of 2-SRS, there exist a single valued choice function $c^{\prime}$ on the binary domain $\Sigma^{\prime}=\Sigma \bigcup\{\{x, y\} \mid x, y \in X\}$ such that:

- $\left(X, \Sigma^{\prime}, c^{\prime}\right)$ is a 'yes' instance of $2-\mathrm{SRS}$, i.e. $\left(X, \Sigma^{\prime}, c^{\prime}\right)$ satisfies WWE, and

- $c^{\prime}$ agrees with $c$ on the domain $\Sigma$, i.e. for all $A \in \Sigma, c(A)=c^{\prime}(A)$.

Now, let us construct such choice function $c^{\prime}$. Consider a pair of alternatives $x, y \in X$ for which $\{x, y\} \notin \Sigma$. If $x \in c\left(S_{i}\right)$ for a class and there exist a choice set $R \in \Sigma$ such that $y \in c(R)$ and $R \subseteq \bigcup_{i} S_{i}$, we impose that $\{y\}=c^{\prime}(\{x, y\})$. Similarly if $y \in c\left(V_{i}\right)$ for a class and there exist a choice set $T \in \Sigma$ such that $x \in c(T)$ and $T \subseteq \bigcup_{i} V_{i}$, we set $\{x\}=$ $c^{\prime}(\{x, y\})$. If none of above two conditions are satisfied, we pick at random an element out of $\{x, y\}$, say $z$ and we set $c(\{x, y\})=\{z\}$. Condition NB-WWE guarantees that for no pair of alternatives $x$ and $y$, we have that both $\{x\}=c(\{x, y\})$ and $\{y\}=c(\{x, y\})$. It is easily verified that the instance $\left(X, \Sigma^{\prime}, c^{\prime}\right)$ satisfies WWE.

The condition NB-WWE can be verified in a polynomial number of steps, hence 2-SRS is in $\mathcal{P}$.

Proposition 3. The decision problem 2-SRS is in $\mathcal{P}$.

Two issues remain, (i) how easy is it to verify $K$-SRS for $K>2$ en (ii) how easy is it to verify $K$-SR for $K>1$. We have the following results. 


\section{Proposition 4.}

- The decision problem $K-S R S$ is $\mathcal{N} \mathcal{P}$-complete for all $K \geq 3$. This result remains if we require the domain to be binary and the rationales to be acyclic (or transitive).

- The decision problem $K-S R$ is $\mathcal{N} \mathcal{P}$-complete for all $K \geq 2$. This result remains if we require the domain to be binary and the rationales to be acyclic.

Proof. It is easily verified that $K$-SR and $K$-SRS are in $\mathcal{N} \mathcal{P}$. For the second part of the proof, we begin with the problem $K$-SRS. The proof uses a reduction from the $\mathcal{N} \mathcal{P}$ complete problem 3SAT. The instance and question of 3SAT are the following:

\section{SAT}

INSTANCE: A list of binary variables $\left\{x_{1}, \ldots, x_{t}\right\}$ and a set of clauses $\left\{C_{1}, \ldots, C_{r}\right\}$ where each clause $\ell=1, \ldots, r$ exists of three literals $l_{1, \ell}, l_{2, \ell}$ and $l_{3, \ell}$. Each literal either equals a certain variable or its negation. The size of the instance is given by the numbers $t$ and $r$.

QUESTION: Does there exist an assignment to the variables $\left\{x_{1}, \ldots, x_{t}\right\}$ (either 1 or $0)$ such that for every clause $C_{\ell}(\ell=1, \ldots, r)$ at least one literal has the value 1 ?

Now we are ready to show that 3SAT is polynomial time reducible to $K$-SRS for $K>3$. Consider an instance of 3SAT with a set of variables $\left\{x_{1}, \ldots, x_{t}\right\}$ and a set of clauses $\left\{C_{1}, \ldots, C_{r}\right\}$. First we create the set of alternatives.

- For each variable $x_{i}, i=1, \ldots, t$, we create two alternatives $a_{i}$ and $\bar{a}_{i}$.

- We create 3 other additional alternatives $v_{1}, v_{2}$ and $q$.

Consider the function $f$ from the set of elements $(k, \ell)(k=1,2,3$ and $\ell=1, \ldots, r)$ to the set of alternatives, $X$, such that $f(k, \ell)=a_{i}$ if the $k$ th literal in the $\ell$ th clause, $C_{\ell}$, equals $x_{i}$ and $f(k, \ell)=\bar{a}_{i}$ if the $k$ th literal in the $\ell$ th clause equals $\left(1-x_{i}\right)$. The choice sets, $\Sigma$ and the choice function, $c$, are given in the following table. Notice that the domain is binary. 


\begin{tabular}{l|c|c|c} 
choice set & choice & range & \\
\hline$\left\{v_{1}, v_{2}\right\}$ & $\left\{v_{1}\right\}$ & & $(1)$ \\
$\left\{v_{1}, q\right\}$ & $\{q\}$ & & $(2)$ \\
$\left\{v_{1}, a_{i}\right\}$ & $\left\{v_{1}\right\}$ & $\forall i=1, \ldots, t$ & $(3)$ \\
$\left\{v_{1}, \bar{a}_{i}\right\}$ & $\left\{v_{1}\right\}$ & $\forall i=1, \ldots, t$ & $(4)$ \\
$\left\{v_{2}, q\right\}$ & $\{q\}$ & & $(5)$ \\
$\left\{v_{2}, a_{i}\right\}$ & $\left\{v_{2}\right\}$ & $\forall i=1, \ldots, t$ & $(6)$ \\
$\left\{v_{2}, \bar{a}_{i}\right\}$ & $\left\{v_{2}\right\}$ & $\forall i=1, \ldots, t$ & $(7)$ \\
$\left\{q, a_{i}\right\}$ & $\left\{a_{i}\right\}$ & $\forall i=1, \ldots, t$ & $(8)$ \\
$\left\{q, \bar{a}_{i}\right\}$ & $\left\{\bar{a}_{i}\right\}$ & $\forall i=1, \ldots, t$ & $(9)$ \\
$\left\{a_{i}, \bar{a}_{i}\right\}$ & $\left\{a_{i}\right\}$ & $\forall i=1, \ldots, t$ & $(10)$ \\
$\left\{a_{i}, a_{j}\right\}$ & $\left\{a_{i}\right\}$ & $\forall i<j ; i, j=1, \ldots, t$ & $(11)$ \\
$\left\{a_{i}, \bar{a}_{j}\right\}$ & $\left\{a_{i}\right\}$ & $\forall i, j=1, \ldots, t$ & $(12)$ \\
$\left\{\bar{a}_{i}, \bar{a}_{j}\right\}$ & $\left\{\bar{a}_{i}\right\}$ & $\forall i<j ; i, j=1, \ldots, t$ & $(13)$ \\
$\left\{v_{1}, v_{2}, a_{i}, q\right\}$ & $\{q\}$ & $\forall i=1, \ldots, t$ & $(14)$ \\
$\left\{v_{1}, v_{2}, \bar{a}_{i}, q\right\}$ & $\{q\}$ & $\forall i=1, \ldots, t$ & $(15)$ \\
$\left\{v_{1}, a_{i}, \bar{a}_{i}, q\right\}$ & $\left\{v_{1}\right\}$ & $\forall i=1, \ldots, t$ & $(16)$ \\
$\left\{v_{2}, a_{i}, \bar{a}_{i}, q\right\}$ & $\left\{v_{2}\right\}$ & $\forall i=1, \ldots, t$ & $(17)$ \\
$\left\{v_{2}, f(1, \ell), f(2, \ell), f(3, \ell), q\right\}$ & $\left\{v_{2}\right\}$ & $\forall \ell=1, \ldots, r$ & $(18)$
\end{tabular}

Obviously, this instance of $K$-SRS can be constructed in polynomial time.

Now, we are ready for the proof. Assume that 3SAT is satisfiable. We show that we can find a sequential rationalization $\left(P_{1}, P_{2}, P_{3}\right)$.

For all $i=1, \ldots, t$ with $x_{i}=1$, set $\left(v_{1}, a_{i}\right) \in P_{1}$ and $\left(v_{2}, \bar{a}_{i}\right) \in P_{1}$. If $x_{i}=0$, we set $\left(v_{1}, \bar{a}_{i}\right) \in P_{1}$ and $\left(v_{2}, a_{i}\right) \in P_{1}$. These are the only elements in $P_{1}$.

For all $i=1, \ldots, t$, set $\left(a_{i}, q\right) \in P_{2}$ and $\left(\bar{a}_{i}, q\right) \in P_{2}$. These are the only elements in $P_{2}$. The elements of $P_{3}$ are listed in the table below:

\begin{tabular}{c|l} 
elements of $P_{3}$ & range \\
\hline$\left(v_{1}, v_{2}\right)$ & \\
$\left(q, v_{1}\right),\left(q, v_{2}\right)$ & \\
$\left(a_{i}, \bar{a}_{i}\right)$ & $\forall i=1, \ldots, t$ \\
$\left(v_{2}, a_{i}\right),\left(v_{2}, \bar{a}_{i}\right)$ & $\forall i=1, \ldots, t$ \\
$\left(v_{1}, a_{i}\right),\left(v_{2}, \bar{a}_{i}\right)$ & $\forall i=1, \ldots, t$ \\
$\left(a_{i}, a_{j}\right),\left(\bar{a}_{i}, \bar{a}_{j}\right)$ & $\forall i<j ; i, j=1, \ldots, t$ \\
$\left(a_{i}, \bar{a}_{j}\right)$ & $\forall i, j=1, \ldots, t$
\end{tabular}

Notice that the relations $P_{1}, P_{2}$ and $P_{3}$ are acyclic and that the rationalization is singlevalued. We could also make them transitive by taking their transitive closure. One can easily verify that these three relations rationalize the instance.

To see the reverse, assume that the instance is a 'yes' instance of $K$-SRS with a rationalization $\left(P_{k}\right)_{k \leq K}$. We need to introduce some new notation. Consider 4 alternatives $a, b, c$ and $d$. We write $a b \unrhd c d$ if:

$$
\min \left\{\min _{i}\left\{(a, b) \in P_{i}\right\} ; K+1\right\} \leq \min \left\{\min _{i}\left\{(c, d) \in P_{i}\right\} ; K+1\right\} .
$$


In other words, we have that $a b \unrhd c d$ if the first rationale in the list that contains $(a, b)$ is earlier than the first rationale that contains $(c, d)$. Similarly, we write $a b \triangleright c d$ if:

$$
\min \left\{\min _{i}\left\{(a, b) \in P_{i}\right\} ; K+1\right\}<\min \left\{\min _{i}\left\{(c, d) \in P_{i}\right\} ; K+1\right\} .
$$

Consider the following lemma:

Lemma 1. If the instance $(X, \Sigma, c)$ is a 'yes' instance of $K$-SRS then for all $i=1, \ldots, t$, either

$$
\left(v_{2} \bar{a}_{i} \triangleright \bar{a}_{i} q\right)
$$

or (exclusively),

$$
\left(v_{2} a_{i} \triangleright a_{i} q\right)
$$

Proof. First of all, from (8) and (9) it follows that there must be a $P_{i}$ and $P_{k}$ in the list such that $\left(a_{i}, q\right) \in P_{i}$ and $\left(\bar{a}_{i}, q\right) \in P_{j}$. From (14) and (15), it follows that:

$$
\left(v_{1} a_{i} \triangleright a_{i} q\right) \quad \text { or } \quad\left(v_{2} a_{i} \triangleright a_{i} q\right)
$$

and

$$
\left(v_{1} \bar{a}_{i} \triangleright \bar{a}_{i} q\right) \quad \text { or } \quad\left(v_{2} \bar{a}_{i} \triangleright \bar{a}_{i} q\right)
$$

A negation of one of these conditions would imply that $\{q\} \neq c\left(\left\{v_{1}, v_{2}, a_{i}, q\right\}\right)$ or $\{q\} \neq$ $c\left(\left\{v_{1}, v_{2}, \bar{a}_{i}, q\right\}\right)$.

From (2) and (5) it follows that there must be a $P_{i}$ and $P_{j}$ such that $\left(q, v_{1}\right) \in P_{i}$ and $\left(q, v_{2}\right) \in P_{j}$. Combined with (16) and (17), it follows that:

$$
\text { not }\left[\left(v_{1} a_{i} \triangleright a_{i} q\right) \quad \text { and } \quad\left(v_{1} \bar{a}_{i} \triangleright \bar{a}_{i} q\right)\right]
$$

and

$$
\operatorname{not}\left[\left(v_{2} a_{i} \triangleright a_{i} q\right) \quad \text { and } \quad\left(v_{2} \bar{a}_{i} \triangleright \bar{a}_{i} q\right)\right]
$$

The proof is completed by taking those combinations that do not lead to a contradiction.

Now, consider a rationalization $\left(P_{i}\right)_{i \leq K}$ of the decision problem $K$-SRS and set $x_{i}=1$ if the first case (C.1) of the lemma is satisfied, i.e. $\left(v_{2} \bar{a}_{i} \triangleright \bar{a}_{i} q\right)$ or equivalently: not $\left(v_{2} a_{i} \triangleright a_{i} q\right)$. On the other hand, we set $x_{i}=0$ if (C.2) is satisfied, i.e. $\left(v_{2} a_{i} \triangleright a_{i} q\right)$ or equivalently: not $\left(v_{2} \bar{a}_{i} \triangleright \bar{a}_{i} q\right)$. Consider a clause $C_{\ell}$ with three literals $l_{1, \ell}, l_{2, \ell}$ and $l_{3, \ell}$, we need to show that for each clause $C_{\ell}$, at least one of its literals hold. Consider the choice:

$$
c\left(\left\{v_{2}, f(1, \ell), f(2, \ell), f(3, \ell), q\right\}\right)=\left\{v_{2}\right\} .
$$


Then it cannot be the case that:

$$
\left(v_{2} f(1, \ell) \triangleright f(1, \ell) q\right) \text { and }\left(v_{2} f(2, \ell) \triangleright f(2, \ell) q\right) \text { and }\left(v_{2} f(3, \ell) \triangleright f(3, \ell) q\right)
$$

As such, there must be at least one literals that has a value of one.

We still need to proof the $\mathcal{N} \mathcal{P}$-completeness for $K$-SR with $K \geq 2$. The reasoning is very similar. Both the set of alternatives $X$ and the domain $\Sigma$ are the same as in the case of $K$-SRS, but there is a slight modification of the choices made from the choice sets:

\begin{tabular}{l|c|c|c} 
Choice set & choice & range & \\
\hline$\left\{v_{1}, v_{2}\right\}$ & $\left\{v_{1}, v_{2}\right\}$ & & $(1)$ \\
$\left\{v_{1}, q\right\}$ & $\left\{v_{1}, q\right\}$ & & $(2)$ \\
$\left\{v_{1}, a_{i}\right\}$ & $\left\{v_{1}\right\}$ & $\forall i=1, \ldots, t$ & $(3)$ \\
$\left\{v_{1}, \bar{a}_{i}\right\}$ & $\left\{v_{1}\right\}$ & $\forall i=1, \ldots, t$ & $(4)$ \\
$\left\{v_{2}, q\right\}$ & $\left\{v_{2}, q\right\}$ & & $(5)$ \\
$\left\{v_{2}, a_{i}\right\}$ & $\left\{v_{2}\right\}$ & $\forall i=1, \ldots, t$ & $(6)$ \\
$\left\{v_{2}, \bar{a}_{i}\right\}$ & $\left\{v_{2}\right\}$ & $\forall i=1, \ldots, t$ & $(7)$ \\
$\left\{q, a_{i}\right\}$ & $\left\{a_{i}\right\}$ & $\forall i=1, \ldots, t$ & $(8)$ \\
$\left\{q, \bar{a}_{i}\right\}$ & $\left\{\bar{a}_{i}\right\}$ & $\forall i=1, \ldots, t$ & $(9)$ \\
$\left\{a_{i}, \bar{a}_{i}\right\}$ & $\left\{a_{i}, \bar{a}_{i}\right\}$ & $\forall i=1, \ldots, t$ & $(10)$ \\
$\left\{a_{i}, a_{j}\right\}$ & $\left\{a_{i}, a_{j}\right\}$ & $\forall i, j=1, \ldots, t$ & $(11)$ \\
$\left\{a_{i}, \bar{a}_{j}\right\}$ & $\left\{a_{i}, \bar{a}_{j}\right\}$ & $\forall i, j=1, \ldots, t$ & $(12)$ \\
$\left\{\bar{a}_{i}, \bar{a}_{j}\right\}$ & $\left\{\bar{a}_{i}, \bar{a}_{j}\right\}$ & $\forall i, j=1, \ldots, t$ & $(13)$ \\
$\left\{v_{1}, v_{2}, a_{i}, q\right\}$ & $\left\{v_{1}, v_{2}, q\right\}$ & $\forall i=1, \ldots, t$ & $(14)$ \\
$\left\{v_{1}, v_{2}, \bar{a}_{i}, q\right\}$ & $\left\{v_{1}, v_{2}, q\right\}$ & $\forall i=1, \ldots, t$ & $(15)$ \\
$\left\{v_{1}, a_{i}, \bar{a}_{i}, q\right\}$ & $\left\{v_{1}\right\}$ & $\forall i=1, \ldots, t$ & $(16)$ \\
$\left\{v_{2}, a_{i}, \bar{a}_{i}, q\right\}$ & $\left\{v_{2}\right\}$ & $\forall i=1, \ldots, t$ & $(17)$ \\
$\left\{v_{2}, f(1, \ell), f(2, \ell), f(3, \ell), q\right\}$ & $\left\{v_{2}\right\}$ & $\forall \ell=1, \ldots, r$ & $(18)$
\end{tabular}

The fact that some of these choices are multi-valued allows us to omit the third rationale $P_{3}$ in the rationalization. We leave the proof of this to the reader.

The results of this section are summarized in the following table:

\begin{tabular}{l|c|c|c} 
Question & $K=1$ & $K=2$ & $K \geq 3$ \\
\hline$K$-RMR & $\mathcal{P}$ & $\mathcal{N} \mathcal{P}$-complete & $\mathcal{N} \mathcal{P}$-complete \\
$K$-SRS & $\mathcal{P}$ & $\mathcal{P}$ & $\mathcal{N} \mathcal{P}$-complete \\
$K$-SR & $\mathcal{N} \mathcal{P}$-complete & $\mathcal{N} \mathcal{P}$-complete & $\mathcal{N} \mathcal{P}$-complete
\end{tabular}

\section{The binary integer programming characterization}

In this section we characterize the decision problems $K$-RMR, $K$-SRS and $K$-SR by means of binary integer programming problems (BIP). BIP problems are a flexible and widely used 
tool to solve NP-complete programs. In principle, any BIP can be solved given enough time. However, from elementary integer programming theory, we know that solving integer programs may become computationally demanding for large problems. Nevertheless, they seem to be performing reasonably well for small to moderate sized problems. Of course, we do not claim that BIP provide the only way to verify the decision problems $K$-RMR and $K$-SR. For example, Apesteguia and Ballester (2005) provide an alternative algorithm that verifies the problem $K$-RMR.

Rationalization by multiple rationales: To begin, we need to translate the instance of the decision problem in a more convenient form. In particular consider two matrixes $\mathcal{A}$ and $\mathcal{C}$ of dimension $n \times m$. The $i-\ell$ th element of matrix $\mathcal{A}$ (resp. $\mathcal{C}$ ) is denoted by $\mathcal{A}(i, \ell)$ (resp. $\mathcal{C}(i, \ell)$ ). We set $\mathcal{A}(i, \ell)$ equal to one if the $i$ th alternative $x_{i}$ is in the $\ell$ th choice set $A_{\ell}$, i.e. $x_{i} \in A_{\ell}$ otherwise we set $\mathcal{A}(i, \ell)$ equal to zero. Similarly, we write $\mathcal{C}(i, \ell)=1$ if $x_{i}$ is chosen from $A_{\ell}$, i.e. $x_{i} \in c\left(A_{j}\right)$, we set $\mathcal{C}(i, \ell)$ equal to zero otherwise.

We introduce $n^{2} \cdot K$ binary variables $\mathcal{P}(i, j, k),(i, j \in\{1, \ldots, n\}$ and $k \in\{1, \ldots, K\})$. The idea is that $\mathcal{P}(i, j, k)$ equals one if the element $(i, j) \in P_{k}$ and $\mathcal{P}(i, j, k)$ equals zero if $(i, j) \notin P_{k}$. Finally we introduce the $m \cdot K$ binary variables $\mathcal{I}(\ell, k)(\ell \in\{1, \ldots, m\}$, $k \in\{1, \ldots, K\})$. The aim is to impose that $\mathcal{I}(\ell, k)$ equals one if and only if $P_{k}$ rationales the choices made from $A_{\ell}$.

We are now ready to give the BIP that characterizes $K$-RMR.

Program: CS-K-RMR: for all $i, j, v=1, \ldots, n, \ell=1, \ldots, m$ and $k=1, \ldots, K$ :

$$
\begin{aligned}
\mathcal{P}(i, j, k)+\mathcal{P}(j, v, k) & \leq 1+\mathcal{P}(i, v, k) \\
\mathcal{P}(i, j, k)+\mathcal{P}(j, i, k) & =1 \quad(i \neq j) \\
\sum_{k=1}^{K} \mathcal{I}(\ell, k) & =1 \\
\mathcal{C}(i, \ell) \cdot \mathcal{I}(\ell, k)+\mathcal{A}(j, \ell) \cdot \mathcal{I}(\ell, k) & \leq 1+\mathcal{P}(i, j, k) \quad(i \neq j)
\end{aligned}
$$

Condition (rmr-1) requires the relations $P_{k}$ to be transitive: if $\left(x_{i}, x_{j}\right) \in P_{k}$ (i.e. $\mathcal{P}(i, j, k)=$ 1 ) and $\left(x_{j}, x_{v}\right) \in P_{k}$ (i.e. $\mathcal{P}(j, v, k)=1$ ), then $\left(x_{i}, x_{v}\right) \in P_{k}$ (i.e. $\mathcal{P}(i, v, k)=1$ ). Condition (rmr-2) imposes the conditions of connectedness and asymmetry on $P_{k}$ : either $\left(x_{i}, x_{j}\right) \in P_{k}$ (i.e. $\mathcal{P}(i, j, k)=1)$ or $\left(x_{j}, x_{i}\right) \in P_{k}$ (i.e. $\left.\mathcal{P}(j, i, k)=1\right)$ but not both. Condition (rmr-3) requires that every choice set $C_{\ell}$ should be rationalized by at least one relation $P_{k}$. Finally, requirement $(\mathrm{rmr}-4)$ states that if $x_{i}$ was chosen from $A_{\ell}$ (i.e. $\mathcal{C}(i, \ell)=1$ ), $x_{j}$ is in $A_{\ell}$ (i.e. $\mathcal{A}(j, \ell)=1)$ and $P_{k}$ rationalizes $A_{\ell}(\mathcal{I}(\ell, k)=1)$, then $\left(x_{i}, x_{j}\right) \in P_{k}$ (i.e. $\left.\mathcal{P}(i, j, k)=1\right)$.

Proposition 5. An instance $(X, \Sigma, c)$ is a 'yes' instance for the decision problem $K-R M R$ if and only if the corresponding program CS-K-RMR has a solution.

Sequential Rationalizability: The BIP corresponding to the sequential rationalizability problem is a bit more involved. As before, we consider the two matrixes $\mathcal{A}$ and $\mathcal{C}$ of dimension $n \times m$ constructed similar to above. 
Again, we introduce $n^{2} \cdot K$ binary variables $\mathcal{P}(i, j, k), i, j \in\{1, \ldots, n\}$ and $k \in$ $\{1, \ldots, K\}$. In addition, we introduce $n \cdot(K+1) \cdot m$ variables $\mathcal{N}(i, k, \ell), k=0, \ldots, K$, $i=1, \ldots, n$ and $\ell=1, \ldots, m$. We will require that $\mathcal{N}(i, k, \ell)=1$ if and only if $x_{i} \in M_{k}\left(A_{\ell}\right)$. Finally, we introduce $n^{2} \cdot K \cdot m$ variables, $\delta(i, j, k, \ell)$ which equals one if either $x_{j} \notin M_{k-1}\left(A_{\ell}\right)$ or $\left(x_{j}, x_{i}\right) \notin P_{k}$.

Program: CS-K-SR: for all $i, j, v=1, \ldots, n ; \ell=1, \ldots, m$ and $k=1, \ldots, K$ :

$$
\begin{aligned}
\mathcal{P}(i, j, k)+\mathcal{P}(j, i, k) & \leq 1 \\
\mathcal{N}(i, 0, \ell) & =\mathcal{A}(i, \ell) \\
\mathcal{N}(i, K, \ell) & =\mathcal{C}(i, \ell) \\
\mathcal{N}(i, k, \ell) & \leq \mathcal{N}(i, k-1, \ell) \\
\mathcal{P}(j, i, k)+\mathcal{N}(j, k-1, \ell)+\mathcal{N}(i, k, \ell) & \leq 2 \\
\mathcal{N}(j, k-1, \ell)+\delta(i, j, k, \ell) & \geq 1 \\
\mathcal{P}(j, i, k)+\delta(i, j, k, \ell) & \geq 1 \\
\sum_{j} \delta(i, j, k, \ell)+\mathcal{N}(i, k-1, \ell)-\mathcal{N}(i, k, \ell) & \leq n
\end{aligned}
$$

Program: CS-K-SRS: for all $i, j, v=1, \ldots, n ; \ell=1, \ldots, m$ and $k=1, \ldots, K$, conditions (sr-1)-(sr-8) are satisfied and in addition:

$$
\sum_{k=1}^{K} \mathcal{P}(i, j, k)+\mathcal{P}(j, i, k) \geq 1 \quad(i \neq j)
$$

Condition (sr-1) imposes asymmetry on the relation $P_{k}$ : not both $\left(x_{i}, x_{j}\right) \in P_{k}$ (i.e. $\mathcal{P}(i, j, k)=1)$ and $\left(x_{j}, x_{i}\right) \in P_{k}$ (i.e. $\left.\mathcal{P}(j, i, k)=1\right)$. Rules (sr-2) and (sr-3) define the sets $M_{0}\left(A_{\ell}\right)=A_{\ell}$ (i.e. $\mathcal{N}(i, 0, \ell)=1$ if and only if $\mathcal{A}(i, \ell)=1$ ) and $M_{K}\left(A_{\ell}\right)=c\left(A_{\ell}\right)$ (i.e. $\mathcal{N}(i, K, \ell)=1$ if and only if $\mathcal{C}(i, \ell)=1)$. Rule (sr-4) implies that when $x_{i} \notin M_{k-1}\left(A_{\ell}\right)$ (i.e. $\mathcal{N}(i, k-1, \ell)=0$ ) then $x_{i} \notin M_{k}\left(A_{\ell}\right)$ (i.e. $\mathcal{N}(i, k, \ell)=0$ ). Rule (sr-5) implies that when $\left(x_{j}, x_{i}\right) \in P_{k}$ (i.e. $\mathcal{P}(j, i, k)=1$ ) and $x_{j} \in M_{k-1}\left(A_{\ell}\right)$ (i.e. $\mathcal{N}(j, k-1, \ell)=1$ ), then $x_{i} \notin M_{k}\left(A_{\ell}\right)$ (i.e. $\left.\mathcal{N}(i, k, \ell)=0\right)$. Condition (sr-6) implies that when $x_{j} \notin M_{k-1}\left(A_{\ell}\right)$ (i.e. $\mathcal{N}(j, k-1, \ell)=0)$ then $\delta(i, j, k, \ell)=1$. Condition (sr-7) implies that when $\left(x_{j}, x_{i}\right) \notin P_{k}$ (i.e. $\mathcal{P}(j, i, k)=0)$, then also $\delta(i, j, k, \ell)=1$. Finally, condition (sr-8) expresses the condition that if for all $j \in X$ either $x_{j} \notin M_{k-1}\left(A_{\ell}\right)$ or $\left(x_{j}, x_{i}\right) \notin P_{k}$ (i.e. $\delta(i, j, k, \ell)=1$ ) and if $x_{i} \in M_{k-1}\left(A_{\ell}\right)$ (i.e. $\mathcal{N}(i, k-1, \ell)=1$ ), then $x_{i} \in M_{k}\left(A_{\ell}\right)$ (i.e. $\left.\mathcal{N}(i, k, \ell)=1\right)$.

For CS-K-SRS, we have the additional condition (srs-9) which states that for all alternatives $x_{i}, x_{j} \in X$ there must be at least one rationale $P_{k}$ such that either $\left(x_{i}, x_{j}\right) \in P_{k}$ (i.e. $\mathcal{P}(i, j, k)=1)$ or $\left(x_{j}, x_{i}\right) \in P_{k}$ (i.e. $\left.\mathcal{P}(j, i, k)=1\right)$.

Proposition 6. An instance $(X, \Sigma, c)$ is a 'yes' instance for the decision problem $K-S R$ if and only if the corresponding program CS-K-SR has a solution. It is a 'yes' instance for the decision problem $K-S R S$ if and only if the corresponding program CS-K-SRS has a solution. 
Simulation Results: In order to illustrate our BIP approach. We compute the power of the decision problems. Here, we follow Bronars (1987) and define power as the probability of rejecting random choice behavior. For the simulation, we need to fix the size of the instance. In particular, we choose $n=6$ and $m=6$, i.e. we took instances with six alternatives and six choice sets. We also need some algorithm to construct the choice sets. We do this by drawing for each alternative $x_{i},(i=1, \ldots, n)$ and each choice set $A_{\ell}$, $(\ell=1, \ldots, m)$ a random number in $[0,1]$. Next we put $x_{i} \in A_{\ell}$ if this random number is greater than some predetermined level $\alpha$ (making sure of course that no choice set is empty). Of course, the lower the value of $\alpha$, the larger the average choice set in the instance. Next, we draw at random an element from each set $A_{\ell}$ which we identify with $c\left(A_{\ell}\right)$. The following table gives the fraction of simulated instances $(X, \Sigma, c)$ that are rejected by the models (1.000 simulations).

\begin{tabular}{cc|c|c|c|c|c} 
Model & $\alpha$ & $K=1$ & $K=2$ & $K=3$ & $K=4$ & $K=5$ \\
\hline RMR & 0.2 & 0.998 & 0.774 & 0.157 & 0.002 & 0.000 \\
& 0.4 & 0.986 & 0.320 & 0.006 & 0.000 & 0.000 \\
& 0.6 & 0.830 & 0.030 & 0.000 & 0.000 & 0.000 \\
& 0.8 & 0.434 & 0.002 & 0.000 & 0.000 & 0.000 \\
\hline SR & 0.2 & 0.998 & 0.906 & 0.802 & 0.783 & 0.780 \\
& 0.4 & 0.96 & 0.559 & 0.332 & 0.302 & 0.292 \\
& 0.6 & 0.748 & 0.244 & 0.147 & 0.139 & 0.138 \\
& 0.8 & 0.362 & 0.114 & 0.101 & 0.099 & 0.099 \\
\hline SRS & 0.2 & 0.998 & 0.914 & 0.802 & 0.783 & 0.780 \\
& 0.4 & 0.986 & 0.593 & 0.334 & 0.302 & 0.292 \\
& 0.6 & 0.83 & 0.267 & 0.147 & 0.139 & 0.138 \\
& 0.8 & 0.434 & 0.117 & 0.101 & 0.099 & 0.099
\end{tabular}

Kalai, Rubinstein, and Spiegler (2002) showed that any instance $(X, \Sigma, c)$ where $X$ has $n$ elements is a 'yes' instance for $(n-1)$-RMR. This fact is reproduced in the last column of the upper part of the table, where we see that the power for 5-RMR is indeed equal to zero. We make a few observations. First of all, the tests are clearly nested where $K$-RMR, $K$-SR and $K$-SRS imply, respectively, $K^{\prime}$-RMR, $K^{\prime}$-SR and $K^{\prime}$-SRS for all $K \leq K^{\prime}$ and $K$-SRS implies $K$-SR. Second, larger choice sets (lower value of alpha) produce higher power. Finally, we see that, except for the case $K=1$, the decision problems $K$-SR and $K$-SRS have higher power than the decision problem $K$-RMR.

Remarks: We briefly discuss three further interesting extensions of the proposed BIP approach: (i) impose or relax certain properties on the rationales, (ii) recovery the underlying rationales and (iii) goodness of fit measures when the instance is not rationalizable.

i The BIP approach is a very flexible took to impose or relax certain conditions on the rationales. If, for example, we want to impose transitivity on the relations $P_{k}$ 
in the rationalization of $K$-SR or $K$-SRS, it suffices to introduce the additional constraint (rmr-1) into program CS-K-SR(S). If we only want to impose the less stringent condition of acyclicity $P_{k}$, we can do this by constructing $n^{2} \cdot K$ additional variables $\mathcal{Q}(i, j, k)$ and imposing the following conditions for all $i, j=1, \ldots, n$ and $k=1, \ldots, K$ :

$$
\begin{aligned}
\mathcal{P}(i, j, k) & \leq \mathcal{Q}(i, j, k) \\
\mathcal{Q}(i, j, k)+\mathcal{Q}(j, v, k) & \leq 1+\mathcal{Q}(i, v, k) \\
\mathcal{Q}(i, j, k)+\mathcal{Q}(j, i, k) & \leq 1 \quad(i \neq j)
\end{aligned}
$$

The idea behind the construction is to construct a transitive and asymmetric relation $Q_{k}$ for which $P_{k} \subseteq Q_{k}$. Condition (sr-9) implies that $P_{k} \subseteq Q_{k}$ (i.e. if $\mathcal{P}(i, j, k)=1$ then $\mathcal{Q}(i, j, k)=1$ ) while condition (sr-10) implies transitivity of $Q_{k}$ and condition (sr-11) implies asymmetry of $Q_{k}$. These extra conditions imply extra structure on the rationales of the decision problem $K-\mathrm{SR}(\mathrm{S})$. Of course, we could also apply these methods to relax certain properties of the rationales for the decision problem $K$ RMR.

- The BIP approach not only verifies whether a certain instance can be rationalized by the different boundedly rational choice models, but it also reconstructs a collection of rationales that lead to this rationalization using the variables $P(i, j, k)$. As such, this approach can in fact be used to reconstruct the underlying features of the model.

- Finally the BIP approach can also be used to compute goodness of fit measures if a certain instance is not rationalizable by the boundedly rational choice model. For example, one could compute the minimal number of asymmetry violations needed in order to rationalize a particular instance.

\section{Descriptive complexity}

In this section, we use our $\mathcal{N} \mathcal{P}$-completeness results to explain some characterization results in the literature. For compactness, we limit ourselves to a fairly intuitive discussion. For a rigorous overview of the theory of descriptive complexity, we refer to the book of Immerman (1999). We also refer to the research of Galambos (2009) who uses descriptive complexity theory together with an $\mathcal{N} \mathcal{P}$-completeness result to discuss certain domain restrictions on the choice functions that are rationalizable by a noncooperative Nash equilibrium.

In order to motivate our discussion, recall the characterization of 2-SRS given by Manzini and Mariotti (2007):

Theorem (Manzini and Mariotti (2007)). An instance $(X, \Sigma, c)$ with a binary domain $\Sigma$ is a 'yes' instance for the decision problem 2-SRS if and only if it satisfies $W W E$ : $c$ is single valued and if $x=c\left(S_{i}\right)$ for a class and $x=c(\{x, y\})$ then $y \neq c(R)$ for all $R \in \Sigma$ with $\{x, y\} \subset R \subseteq \bigcup_{i \leq T} S_{i}$. 
From a computational complexity point of view, this is a 'nice' characterization. In particular, it is quite easy to envisage the way in which we should verify the property WWE: for any two elements $x$ and $y$ from $X$, look if $x \in c(\{x, y\})$. If this is the case, collect the choice sets $S_{i}$ for which $x \in c\left(S_{i}\right)$ and the choice sets $V_{j}$ for which $y \in c\left(V_{j}\right)$ and verify that for all $j$ it is not the case that $V_{j} \subseteq \bigcup_{i} S_{i}$. This algorithm would decide in polynomial time whether a particular instance satisfies WWE.

In the theory of mathematical logic, properties (sentences) are not categorized by their computational complexity but by the logical language that is used to express them. One of the most elementary languages is called first order logic. Sentences in first order logic are composed of variables (in our case these are the alternatives in $X$ and choice sets in $\Sigma$ ), constant symbols, relational symbols (the rationales " $P_{i}$ " and equality sign "="), boolean connectives (and, " $\wedge$ ", or, " $\vee$ " and not, " $\neg "$ ") and quantifiers (for all, " $\forall$ " and there exists, " $\exists$ ") which are restricted over the set of variables. It is easy to see that the property WWE can be expressed in first order logic.

Descriptive complexity theory investigates the relationship between the computational complexity of a certain problem and the logical language necessary to characterize this problem. Consider a decision problem and let $\mathcal{I}$ be the instances of this problem. The set $\mathcal{I}$ can then be partitioned into the set of instances that are a 'yes' instance of the decision problem $\mathcal{I}_{Y}$ and the instances that correspond to a 'no' instance $\mathcal{I}_{N}$. Then, one can try to find a property $T$ that characterizes the instances in $\mathcal{I}_{Y}$. Descriptive complexity asks how hard it is to express this property in a certain logic. As an example, consider the decision problem 2-SRS on the set of binary domains. This decision problem is in the class $\mathcal{P}$. A instance $(X, \Sigma, c)$ is in the class $\mathcal{I}_{Y}$ of 2-SRS if and only if it satisfies the property WWE, and this property is expressible in first order logic.

One of the most important results in descriptive complexity theory gives the equivalence between the decision problems in $\mathcal{P}$ and the instances that can be characterized using properties expressible in first order logic augmented with the least fixed point operator. The least fixed point operator adds to first order logic the power to define new relations by induction. The most relevant example of the least fixed point operator, for choice theory, is the transitive closure operator. ${ }^{7}$ The transitive closure of a relation cannot be expressed using only first order logic but it can nevertheless be computed in polynomial time (using for example Warshall (1962)'s algorithm).

Theorem 2 (Immerman-Vardi theorem (Immerman, 1982; Vardi, 1982)). Over ordered structures, the set of 'yes' instances to a decision problem can be characterized by a property expressible in first order logic together with the least fixed point operator if and only if the decision problem is in the class $\mathcal{P}$.

The requirement of ordered structures does not need to concern us here. It merely requires that there is some kind of ordering over the variables in the language. Of course

\footnotetext{
${ }^{7}$ The transitive closure operator $T$ (.) can be defined inductively by constructing the relations $P_{t}(t=$ $1, \ldots, T, T+1, \ldots$,$) where P_{1}=P$ and $(x, y) \in P_{t}$ if $(x, y) \in P_{t-1}$ or if there exist a $z$ such that $(x, z) \in P_{t-1}$ and $(z, y) \in P_{t-1}$. The transitive closure $T(P)$ of the relation $P$ is then the smallest relation $P_{T}$ such that $P_{T}=P_{T+1}$.
} 
every computational language needs an ordering over its inputs in order to be able to read these inputs and perform computations on them but first order logic alone is not strong enough to define this ordering on its own variables. As such, we need this additional requirement

Now, let us have a look at the property of 3-SRS. For a (multi-valued) choice function $c$ on a domain $\Sigma$, denote by $c(\Sigma)$ the domain:

$$
c(\Sigma)=\{A \subseteq X \mid \exists B \in \Sigma, A=c(B)\} .
$$

Manzini and Mariotti (2007) give the following characterization of 3-SRS:

Theorem 3. An instance $(X, \Sigma, c)$ is a 'yes' instance of the decision problem 3-SRS if and only if $c$ is single valued and there exists a choice function $c^{*}$ on the domain $\Sigma$ such that:

1. $c\left(c^{*}(A)\right)=c(A)$ for all $A \in \Sigma$,

2. $\left\{X, c^{*}(\Sigma), c\right\}$ is a 'yes' instance of 2-SRS,

3. if $x \in c^{*}(A)$ for some $A \in \Sigma$, then $x \in c^{*}(\{x, y\})$ for all $y \in A$.

In contrast to 2-SRS (i.e. property WWE), this characterization is not so 'nice'. In particular, it is not at all obvious how one begins at verifying whether a given choice function satisfies it. ${ }^{8}$ The problem lies in the fact that we must show the existence of a certain choice function $c^{*}$ and that in general the size of the collection of choice functions is very big (exponential). Going back to a logical perspective, the problem is that for this decision problem, the characterization of the 'yes' instances is not expressed in first order logic (with the least fixed point property). In fact, the logic used to express this theorem is given by the language of existential second order logic. Existential second order logic is defined by extending first order logic with the power to condition on the existence of higher order elements like relations or functions. This is exactly what theorem 3 does. It conditions on the existence of the choice function $c^{*}$ (There exists a choice function $c^{*}$ on $\Sigma$ such that...). This leads us to another important theorem in descriptive complexity theory.

Theorem 4 (Fagin (1974)'s theorem). The set of 'yes' instances to a decision problem can be characterized by properties expressible in existential second order logic if and only if the decision problem is in the class $\mathcal{N} \mathcal{P}$.

Combining Fagin's theorem with the Immerman-Vardi theorem, we derive following result.

Corollary 1. Unless $\mathcal{P}=\mathcal{N} \mathcal{P}$ it is not possible to characterize the set of 'yes' instances of an $\mathcal{N} \mathcal{P}$-complete decision problem using only first order logic and the least fixed point operator. In particular, this means that...

\footnotetext{
${ }^{8}$ Interestingly, Manzini and Mariotti (2007) provide such an algorithm, albeit with exponential time complexity.
} 
- ... unless $\mathcal{P}=\mathcal{N P}$ it is not possible to characterize the set of 'yes' instances of the decision problem $K-R M R$ (with $K \geq 2$ ) using only first order logic and the least fixed point operator.

- ... unless $\mathcal{P}=\mathcal{N P}$ it is not possible to characterize the set of 'yes' instances of the decision problem $K$-SRS (with $K \geq 3$ ) using only first order logic and the least fixed point operator.

The above corollary explains why theorem 3 is stated in second order existential logic. If it were possible to give an equivalent characterization expressible in first order logic (together with the least fixed point property) we would have shown that $\mathcal{P}=\mathcal{N} \mathcal{P}$.

Corollary 1 might also explains other findings in the literature. Recently, Bossert, Sprumont, and Suzumura (2005) characterized the instances $(X, \Sigma, c)$ that are maximal element rationalizable by a binary relation and the instances that are maximal element rationalizable by a relation that satisfies one or several of the following properties: completeness, reflexivity, acyclicity, quasi-transitivity, consistency and transitivity.

For an instance $(X, \Sigma, c)$, define:

$$
\mathcal{A}_{c}=\{(A, y) \mid A \in \Sigma \text { and } y \in A-c(A)\} .
$$

and

$$
\mathcal{F}_{c}=\left\{f: \mathcal{A}_{c} \rightarrow X \mid f(A, y) \in A \text { for all }(A, y) \in \mathcal{A}_{C}\right\} .
$$

The set $\mathcal{A}$ collects the pairs $(A, y)$ such that $A$ is a choice set and $y$ is not chosen from $A$ and the functions in $\mathcal{F}_{c}$ assign to every pair $(A, y)$ in $\mathcal{A}$ an element in $A$. The interpretation is that $f(A, y)$ is an alternative in $A$ that can be used to prevent $y$ from being chosen. Observe that the cardinality of $\mathcal{F}_{c}$ is exponential in the size of the instance.

Bossert, Sprumont, and Suzumura (2005) discuss and defend in great length the fact that most of their characterizations involve existential clauses. Consider the first of these characterizations:

Theorem 5 (M-RAT, Bossert, Sprumont, and Suzumura (2005)). Consider an instance $(X, \Sigma, c)$. There exist an (asymmetric) binary relation $P$ such that for all $A \in \Sigma$ :

$$
c(A)=\mathcal{M}(A, P),
$$

if and only if there exist an $f \in \mathcal{F}_{c}$ such that:

- For all $(A, y) \in \mathcal{A}_{c}$, for all $T \in \Sigma$ and for all $x \in T$ :

$$
f(A, y)=x \rightarrow y \notin c(T) .
$$

- For all $(A, y),(T, x) \in \mathcal{A}_{c}$,

$$
f(A, y)=x \rightarrow f(T, x) \neq y
$$


Given above discussion, the problem does not really seems to be the existential clause, but the fact that the characterization of M-RAT is stated in existential second order logic (there exist a function $f \in \mathcal{F}_{c}$ such that...). Confronted with the observation that the authors have not found an easier characterization and in the light of corollary 1 one is easily led to the conjecture that the decision problem M-RAT (which is equal to the decision problem 1 -SR) is $\mathcal{N} \mathcal{P}$-complete. Indeed,

Proposition 7. The following decision problem is $\mathcal{N} \mathcal{P}$-complete:

\section{M-RAT (1-SR)}

QUESTION: Given an instance $(X, \Sigma, c)$, does there exist an (asymmetric) relation $P$ such that for all $A \in \Sigma, c(A)=\mathcal{M}(A, P)$ ?

Proof. Membership in $\mathcal{N} \mathcal{P}$ is easily verified. For the second part, we consider a reduction from 3SAT. Consider an instance of 3SAT given by the variables $\left\{x_{1}, \ldots, x_{t}\right\}$ and set of clauses $\left\{C_{1}, \ldots, C_{r}\right\}$. We now construct the instance of M-RAT:

- for each variable $x_{i}$ we construct two alternatives $a_{i}$ and $\bar{a}_{i}$.

- we construct three additional alternatives $d$ and $c$ and $q$.

Consider the function $f(k, \ell)(k=1,2,3, \ell=1, \ldots, m)$ such that $f(k, \ell)=a_{i}$ if the $k$ th literal in the $\ell$ th clause is given by $x_{i}$ and let $f(k, \ell)=\bar{a}_{i}$ if the $k$ th literal in the $\ell$ th clause is given by $\left(1-x_{i}\right)$. The following table gives the choice sets and choices:

\begin{tabular}{l|c|c|c} 
choice set & choice & range & \\
\hline$\left\{q, a_{i}\right\}$ & $\left\{q, a_{i}\right\}$ & $i=1, \ldots, t$ & $(1)$ \\
$\left\{q, \bar{a}_{i}\right\}$ & $\left\{q, \bar{a}_{i}\right\}$ & $i=1, \ldots, t$ & $(2)$ \\
$\{q, c\}$ & $\{q, c\}$ & & $(3)$ \\
$\left\{d, a_{i}\right\}$ & $\{d\}$ & $i=1, \ldots, t$ & $(4)$ \\
$\left\{d, \bar{a}_{i}\right\}$ & $\{d\}$ & $i=1, \ldots, t$ & $(5)$ \\
$\{d, c\}$ & $\{d, c\}$ & & $(6)$ \\
$\left\{q, a_{i}, \bar{a}_{i}, c\right\}$ & $\{q\}$ & $i=1, \ldots, t$ & $(7)$ \\
$\{d, f(1, \ell), f(2, \ell), f(3, \ell), c\}$ & $\{d\}$ & $\ell=1, \ldots, r$ & $(8)$
\end{tabular}

Consider a 'yes' instance of 3SAT. If $x_{i}=1$ we set $\left(\bar{a}_{i}, a_{i}\right) \in P,\left(a_{i}, c\right) \in P$ and $\left(c, \bar{a}_{i}\right) \in P$ if $x_{i}=0$, we set $\left(a_{i}, \bar{a}_{i}\right) \in P,\left(\bar{a}_{i}, c\right) \in P$ and $\left(c, a_{i}\right) \in P$.

Further set for all $i=1, \ldots, t,\left(d, a_{i}\right),\left(d, \bar{a}_{i}\right) \in P$. It is easy to see that this relation provides a 'yes' instance for M-RAT.

On the other hand, let $P$ be a solution to M-RAT. Now for all $i$, either $\left(\bar{a}_{i}, a_{i}\right),\left(a_{i}, c\right),\left(c, \bar{a}_{i}\right) \in$ $P$ or (exclusive) $\left(a_{i}, \bar{a}_{i}\right),\left(\bar{a}_{i}, c\right),\left(c, a_{i}\right) \in P$ (by (1)-(3), (7) and asymmetry of $P$ ). If the first is the case, we set $x_{i}=1$. If the second is the case, we set $x_{i}=0$. It is easy to see that this provides a 'yes' instance of 3SAT (by (8)).

Verifying whether the other rationalizability problems characterized by Bossert, Sprumont, and Suzumura (2005) are also $\mathcal{N} \mathcal{P}$-complete is left for future research. 
Remark: We conclude this section with one last remark. In this section we judged the 'niceness' of certain characterizations by the logical language in which they are expressed. Of course, we do not claim that this is the only criteria to evaluate the attractiveness of a certain characterization. Characterizations can also be of importance because they provide more insights into the underlying structure of the problem, because they approach the same problem from a different angle, because they emphasize similarities with other well known problems, etc. Even from a more computational point of view, a different characterizations may lead to considerable savings in computing time.

\section{Concluding Discussion}

We have characterized the computational complexity of two boundedly rational choice models: the rationalization by multiple rationales and the sequential rationalization by multiple rationales. We found that in most cases, these models are difficult to verify $(\mathcal{N} \mathcal{P}$ complete). Although these results could be seen as a drawback for future research, this does not have to be the case. We give a few guidelines.

First, although, the $\mathcal{N} \mathcal{P}$ completeness results indicate that it might be computationally infeasible to verify, for large instances, whether they satisfy the two boundedly rational choice models this only shows that empirical applications should concentrate on collecting small to moderate sized instances. In particular, empirical research should focus on collecting a large sample of small instances rather than a small sample of large instances.

Second, even for large instances it is still possible to use heuristics that give a quick (but possibly inconclusive) answer to whether or not the data set satisfies the boundedly rational choice models.

Finally, our results impose very modest domain restrictions. As such, it might be the case that certain choice domains (for example, a domain that is closed under unions, intersections or complements) do allow for efficient, polynomial time, verification.

\section{References}

Apesteguia, J., Ballester, M., 2010. The computational complexity of rationalizaing behavior. Journal of Mathematical Economics 46, 356-363.

Apesteguia, J., Ballester, M. A., 2005. Minimal books of rationales. Tech. Rep. 0501, Universidad Publica de Navarra.

Baron, R., Durieu, J., Haller, H., Savani, R., Solal, P., 2008. Good neighbors are hard to find: Computational complexity of network formation. Review of Economic Design 12, $1-19$.

Baron, R., Durieu, J., Haller, H., Solal, P., 2004. Finding a Nash equilibrium in spatial games is an NP-complete problem. Economic Theory 23, 445-454. 
Bossert, W., Sprumont, Y., Suzumura, K., 2005. Maximal-element rationalizability. Theory and Decision 58, 325-350.

Brandt, F., Fisher, F., 2008. Computing the minimal covering set. Mathematical Social Sciences 56, 254-268.

Brandt, F., Fisher, F., Harrenstein, P., Mair, M., 2010. A computational analysis of the tournament equilibrium set. Social Choice and Welfare 34, 597-609.

Bronars, S. G., 1987. The power of nonparametric tests of preference maximization. Econometrica 55, 693-698.

Cechlarova, K., Hajdukova, J., 2002. Computational complexity of stable partitions with B-preferences. International Journal of Game Theory 31, 353-364.

Cherchye, L., Demuynck, T., De Rock, B., 2009. Testable implications of general equilibrium models: an integer programming approach. Center for Economic Studies - Discussion papers ces09.14, Katholieke Universiteit Leuven, Centrum voor Economische Studin.

Chu, F., Halpern, J., 2001. On the NP-completeness of finding an optimal strategy in games with common payoffs. International Journal of Game Theory 30, 99-106.

Conitzer, V., Sandholm, T., 2008. New complexity results about Nash equilibria. Games and Economic Behavior 63, 621-641.

Fagin, R., 1974. Complexity of Computation, SIAM-AMS Proceedings 7. Ch. Generalized First-Order Spectra and Polynomial-Time Recognizable Sets.

Fang, Q., Zhu, S., Cai, M., Deng, X., 2002. On computational complexity of membership test in flow games and linear production games. International Journal of Game Theory $31,39-45$.

Galambos, A., 2009. The complexity of nash rationalizability. Tech. rep., Lawrence University.

Garey, M. R., Johnson, D. S., 1979. Computers and Intractability. Bell Telephone Laboratories, Inc.

Gilboa, I., Zemel, E., 1989. Nash and correlated equilibria: Some complexity considerations. Games and Economic Behavior 1, 80-93.

Hudry, O., 2009. A survey on the complexity of tournament solutions. Mathematical Social Sciences 57, 292-303.

Immerman, N., 1982. Upper and lower bounds for first-order expressibility. Journal of Computer and System Sciences 25, 76-98. 
Immerman, N., 1999. Descriptive Complexity. Sprimger-Verlag.

Kalai, G., Rubinstein, A., Spiegler, R., 2002. Rationalizing choice functions by multiple rationales. Econometrica 70, 2481-2488.

Kalyanaraman, S., Umans, C., 2008. Lecture notes in Computer Science: Algorithms and Computation. Springer, Ch. The Complexity of Rationlizing Matchings, pp. 171-182.

Kroll, E. B., Vogt, B., 2008. The relevance of irrelevant alternatives: An experimental investigation of risky choices. Tech. Rep. 28, Otto-von-Guericke-University.

Loomes, G., Starmer, C., Sugden, R., 1991. Observing violations of transitivity by experimental methods. Econometrica 59, 425-439.

Loomes, G., Taylor, C., 1992. Non-transitive preferences over gains and losses. Economic Journal 102, 357-365.

Luce, D. R., Raiffa, H., 1957. Games and Decisions: Introduction and Critical Survey. Wiley.

Manzini, P., Mariotti, M., 2007. Sequentially rationalizable choice. American Economic Review 97, 1824-1839.

Procaccia, A. D., Rosenschein, J. S., 2008. On the complexity of achieving proportional representation. Social Choice and Welfare 30, 353-362.

Richter, M. K., 1966. Revealed preference theory. Econometrica 34, 635-645.

Roelofsma, P. H. M., Read, D., 2000. Intransitive intertemporal choice. Journal of Behavioral Decision Making 13, 161-177.

Seidl, C., Traub, S., 1996. Rational choice and the relevance of irrelevant alternatives. Tech. Rep. 1996-91, Tilburg Universit.

Suzumura, K., 1983. Rational Choice, Collective Decisions, and Social Welfare. Cambridge University Press.

Szpilrajn, E., 1930. Sur l'extension de l'ordre partiel. Fundamentae Mathematicae 16, 386389.

Talla Nobibon, F., Cherchye, L., De Rock, B., Sabbe, J., Spieksma, F. C. R., 2010. Heuristics for deciding collectively rational consumption behavior. Computational Economics forthcoming.

Tversky, A., 1969. Intransitivity of preferences. Psychological Review 76, 31-48.

Vardi, M. Y., 1982. Proceedings of the 14th ACM Symposium on Theorey of Computing. Ch. The Complexity of Relational Quiry Languages. 
Warshall, S., 1962. A theorem of boolean matrices. Journal of the American Association of Computing Machinery 9, 11-12.

Woeginger, G. J., 2003. Banks winners in tournaments are difficult to recognize. Social Choice and Welfare 20, 523-528. 